\title{
Self-Assembled Core-Shell Poly(Ethylene Glycol)-POSS Nanocarriers for Drug Delivery
}

\author{
Kyu-Oh Kim¹, Byoung-Suhk Kim², Ick-Soo Kim² \\ ${ }^{1}$ Department of Bioscience and Textile Technology, Shinshu University, Ueda, Japan; ${ }^{2}$ Nano Fusion Technology Research Group, \\ Faculty of Textile Science and Technology, Shinshu University, Ueda, Japan. \\ Email: kbsuhk@yahoo.com, kim@shinshu-u.ac.jp
}

Received April 18 ${ }^{\text {th }}, 2011$; revised May $5^{\text {th }}, 2011$; accepted May $15^{\text {th }}, 2011$.

\begin{abstract}
In this work, novel nanostructured core-shell poly (ethylene glycol) (PEG)-polyhedral oligosilsesquioxane (POSS) nanoparticles were used to encapsulate insulin as new drug delivery carriers. The morphologies, particle size and $\zeta$ potential of the pure nanostructured core-shell PEG-POSS and the corresponding insulin-loaded PEG-POSS nanoparticles were investigated by transmission electron microscopy (TEM) and laser diffraction particle sizer. TEM analysis demonstrated that pure and insulin-loaded self-assembled PEG-POSS nanoparticles were of spherical shape with core-shell nanostructure, and were well-dispersed and uniform in size distribution. Insulin release test showed that insulin was well-protected inside PEG-POSS nanoparticles at gastric $p H$ for $2 \mathrm{hrs}$, and was released at intestinal $\mathrm{pH}$ ( $\mathrm{pH}$ 6 - 7) where the absorption and activation of the drug are necessary. We therefore believe that such nanostructured PEG-POSS nanoparticles could be useful as a potential carrier for insulin drug delivery systems.
\end{abstract}

Keywords: Self-Assembly, Amphiphilic, Nanoparticles, POSS, Insulin, Oral Delivery

\section{Introduction}

In a recent year, nanotechnology has been utilized to develop new therapies and next generation nanosystems for "smart" drug delivery [1]. A variety of organic/inorganic nanomaterials and devices have been often used as delivery vehicle to enhance the therapeutic activity by prolonging drug half-life, improving solubility of hydrophobic drugs, reducing potential immunogenicity, and/or releasing dugs in a sustained or stimuli-triggered fashion. Insulin was known to treat diabetic. Current treatment methods involve regular injections of insulin, which can be both painful and inconvenient, thus leading to low patient compliance [2]. In order to overcome this problem, the oral route is considered to be the most convenient and comfortable means of drug administration for patients. However, oral administration of hydrophilic macromolecules such as peptide/protein drugs is encountered with many difficulties as the drugs have to confront various major barriers in the gastrointestinal (GI) tract. Firstly, peptide/protein drugs get denatured readily by low $\mathrm{pH}$ of gastric medium in the stomach. Secondly, different digestive enzymes in the stomach and small intestine may lead to degradation of peptide/protein drugs [3]. A new carrier system is therefore required to protect pep- tide/protein drugs from the harsh environment in the GI tract.

We have recently developed the organic/inorganic hybrid materials, such as amphiphilic poly(ethylene glycol) (PEG)-polyhedral oligosilsesquioxane (POSS) and poly (vinyl alcohol) (PVA)-POSS hybrids incorporating POSS macromers onto chain-ends or polymer backbone as pendent groups, respectively [4-7]. Thanks to their amphiphilic properties (here, POSS is hydrophobic, PEG and PVA hydrophilic), it can be expected that those POSS-containing polymers can form the micelles by tailoring the composition ratio in polymers and solvent polarity, and this has important implications for drug delivery systems. For instance, we reported [8] that PVAPOSS hybrid showed unagglomerated nanoparticles within a diameter range of $60-90 \mathrm{~nm}$, as confirmed by atomic force microscopy (AFM) and bio-transmission electron microscope (bio-TEM). The prepared nanoparticles were found to improve the control release of anticancer drug; paclitaxel as a model drug. However, there are few reports on the solution properties and micelles/nanoparticles formation of POSS-based polymeric materials for drug delivery system [9-11]. In this report, we prepare the hybrid core-shell nanostructured particles 
composed of POSS as a hydrophobic inner core and PEG as a hydrophilic outer shell by using dialysis approach, and propose novel drug delivery carriers for protein drugs.

\section{Experimental Section}

\subsection{Materials}

Poly(ethylene glycol) $(\mathrm{PEG})$ (molecular weight $=3.4 \mathrm{kDa}$, Aldrich) was purified by repeating twice the process of precipitation into $n$-hexane from chloroform solution. Isocyanatopropyldimethylsilylcyclohexyl-polyhedral oligosilsesquioxane (POSS macromer) was purchased from Tomen Plastics Co., Japan. Dibutyl tin dilaurate (DBTDL; Aldrich, 95\% purity) as a catalyst for urethane formation was used as received. Amphiphilic PEG3.4k- POSS telechelic studied herein was synthesized by direct urethane linkage between the diol end groups of PEG homopolymers and the monoisocyanate group of POSS macromers as catalyzed by DBTDL. ${ }^{1} \mathrm{H}-\mathrm{NMR}$ results confirmed that the amphiphilic PEG3.4k-POSS telechelic was successfully prepared. Evidence for the formation of urethane linking groups comes from the emergence of a weak proton signal at about $4.26 \mathrm{ppm}$, accompanied by the disappearance of a proton signal of the $-\mathrm{CH}_{2}-\mathrm{NCO}$ group (3.15 ppm). That is, the level of incorporation of POSS macromers in the amphiphilic PEG3.4k-POSS telechelic could be determined quantitatively by the monitoring of the resonances for the cyclohexyl groups of POSS macromers. A degree of end functionalization was found to about 2.1, indicating quite consistent with the feed ratio. The detailed synthesis and characterization are described in our previous reports $[4,12]$. Toluene was dried over $\mathrm{CaH}_{2}$ and then distilled under nitrogen prior to use. A sample of $100 \mathrm{mg}$ of insulin, from bovine pan- creas (activity: $\geq 25$ USP units/mg, secondary activity: 2500 units) were purchased from Sigma-Aldrich. All chemicals were of analytical purity or higher quality and were used without further purification.

\subsection{Preparation of Insulin-Loaded Hybrid PEG-POSS Particles}

Insulin solution was prepared by dissolving $100 \mathrm{mg}$ of insulin in $10 \mathrm{ml}$ of $0.01 \mathrm{~N} \mathrm{HCl}$ solution. Insulin encapsulation was carried out on the basis of self assembly process. Briefly, $40 \mathrm{mg}$ of PEG3.4k-POSS was fully dissolved in $10 \mathrm{ml}$ of THF. Then, $0.5,0.9,1.3 \mathrm{ml}$ insulin solutions was added dropwise into the PEG3.4k-POSS solutions, respectively. The mixture was poured into dialysis tube (spectra/Por 6, MWCO: $3.5 \mathrm{kD}$ ) and dialyzed against distilled water at room temperature under magnetic stirring for 1 day. Afterwards, the distilled water was exchanged at least 3 times in order to remove the
THF and $\mathrm{HCl}$ residues. To determine the loading efficiency of insulin in hybrid PEG-POSS nanoparticles, the amount of free insulin in supernatants was assayed by UV-vis spectrophotometry. The drug loading efficiency was calculated using the equations listed below [13,14].

$$
\begin{aligned}
& \text { Loading Efficiency }(\%) \\
& \text { total amount of insulin added } \\
& =\frac{- \text { amount of free insulin }}{\text { total amount of insulin added }} \times 100 \%
\end{aligned}
$$

\subsection{Characterization}

Particle size and $\zeta$ potential of pure and insulin-loaded PEG-POSS nanoparticles were measured depending on $\mathrm{pH}$ of the mixture by laser diffraction particle sizer (Nano-ZS, Malvern Instrument Ltd., UK). FI-IR analysis of the prepared nanaoparticles and insulin was carried out using an IRPrestige-21 (Shimadzu Co., Japan) Transmission Electron Microscopy (TEM) images were taken out on a JEM-2100 LaB6 microscope (JEOL, Japan) operating at an accelerating voltage of $160 \mathrm{kV}$ to observe the morphologies of the obtained pure and insulin-loaded PEG-POSS nanoparticles. The insulin-loaded PEG-POSS nanoparticles dispersed in water was directly dropped onto a carbon-coated copper grid (200-A mesh, Nissin EM Co., Ltd.), followed by drying at room temperature. Finally, the samples were dried in vacuum oven. The average particle size was determined from the TEM micrographs using an image analysis software (Image J, National Institutes of Health, Bethesda, U.S.).

\section{4. pH Effects on Insulin Release from PEG-POSS Nanoparticles}

The $\mathrm{pH}$ of the insulin-loaded nanoparticle suspensions was varied at the ranging from $\mathrm{pH} 2.5$ to $\mathrm{pH} 7.4$ to study the release behaviors of the insulin from PEG-POSS nano-particles at room temperature. The insulin-loaded PEG-POSS nanoparticles treated with centrifugation 3000 $\mathrm{rpm} / 15 \mathrm{~min}$ were placed into $10 \mathrm{ml}$ of PBS buffer solution $\left(\mathrm{pH} \mathrm{2.5)}\right.$ and incubated at $37^{\circ} \mathrm{C}$ for $2 \mathrm{hrs} .0 .5 \mathrm{~mL}$ of the supernatants, which was isolated by centrifugation (2000 rpm/1 min), was taken by every twenty minutes for the measurement of the released amount of insulin from PEG-POSS nanoparticles and replaced by fresh medium. Afterwards, the same insulin-loaded PEGPOSS nanoparticles were again isolated by centrifugation (3500 $\mathrm{rpm} / 15 \mathrm{~min}$ ) and were transferred to $10 \mathrm{~mL}$ of phosphate buffer at $\mathrm{pH} 7.4$ and incubated at $37^{\circ} \mathrm{C}$ for 3 hrs. The released amount of insulin from PEG-POSS nanoparticles were monitored by UV-vis spectrophotometry. A plot of cumulative release with time was reported, where 


$$
\text { Cumulative release }(\%)=\frac{A_{t}}{A_{M A X}} \times 100 \%
$$

where $A_{t}$ is the absorbance of the characteristic peak at $275 \mathrm{~nm}\left(\varepsilon=5.53 \mathrm{mM}^{-1} \cdot \mathrm{cm}^{-1}\right)[15]$ at time $t$, and $A_{M A X}$ is the maximum absorbance of this peak.

\section{Results and Discussion}

In our previous paper [4-7], we have reported that the synthesized organic/inorganic PEG-POSS and PVAPOSS hybrid materials were found to be amphiphilic, and thereby resulted in self-assembling into core-shell nanostructured micelles with hydrophobic inner-core (POSS moieties) and hydrophilic outer-shell (PEG or PVA moieties) in aqueous media. Therefore, hydrophobic drugs, such as insulin used in this work can be easily entrapped into the core. Scheme 1 shows the schematic illustration of self-assembling process, which leads to self-aggregation into core-shell nanostructured particles (i.e., flower-like micelles) encapsulating insulin inside the hydrophobic core in aqueous solution. Here, it is worth mentioning that the formation of self-assembled nanostructures using POSS-PEG-POSS telechelic (ABA triblock copolymers) depends on two competing forces: the entropy loss due to loop formation of the central block in the micelle corona and the interfacial energy penalty that accompanies the transfer of insoluble block from the micelle corona to the solution [16].

\subsection{Characterization of Insulin-Loaded PEG-POSS Nanoparticles}

Figure 1 shows TEM images of pure and insulin-loaded self-assembled PEG-POSS nanoparticles. It can be clearly seen that insulin-loaded PEG-POSS nanoparticles were of spherical shape with core-shell nanostructure, and were well-dispersed and uniform in size distribution unlike pure PEG-POSS nanoparticles. The size of pure selfassembled PEG-POSS nanoparticles was about $15.9 \pm$ $1.3 \mathrm{~nm}$, while the insulin-loaded self-assembled PEGPOSS nanoparticles (loading content $\sim 0.9 \mathrm{mg}$ ) were 330 $\pm 80 \mathrm{~nm}$, as measured by TEM analysis, suggesting successful encapsulation of insulin molecules into a hydrophobic core. In addition, the loading efficiency (LE) of insulin in the insulin-loaded PEG-POSS nanoparticles with an added insulin contents of 5, 9, $13 \mathrm{mg}$ was found to be $52.6 \%, 70.5 \%$ and $76.5 \%$, indicating that PEGPOSS nanoparticles have good loading capability of hydrophobic drug, insulin [17,18]. In addition, FT-IR analysis demonstrated the existence of insulin in insulinloaded PEG-POSS nanoparticles (Figure 2). The characteristic peaks at $1651 \mathrm{~cm}^{-1}$ and $1531-1514 \mathrm{~cm}^{-1}$ region were observed respectively, corresponding to $\mathrm{C}-\mathrm{N}$ stretching and $\mathrm{N}-\mathrm{H}$ bending modes of amide II region in pristine insulin [19]. The Si-O-Si peak in the insulinloaded PEG-POSS nanoparticles was shifted toward higher wavenumber, compared to pure PEG-POSS nanoparticles. This suggests that there are strong interacttions between insulin and PEG-POSS segments [20].

\section{2. pH Effects on Insulin-Loaded PEG-POSS Nanoparticles}

The $\zeta$-potential and size of the obtained hybrid nanoparticles was investigated at various $\mathrm{pH}$ and the results were shown in Figure 3. The $\zeta$-potential of pure PEG-POSS nanoparticles was close to constant zero $\zeta$-potential values as increasing $\mathrm{pH}$, whereas the insulin-loaded PEG-POSS nanoparticles obviously exhibited increased negative charges, presumably due to ionized carboxyl groups in an encapsulated insulin (Figure 3(a)) [21]. Moreover, it is also expected that such negative charged nanoparticles may be less aggregated and show good dispersion-stabilities because of an electrostatic repulsion. Therefore, the resultant negatively charged insulin-loaded PEG-POSS nanoparticles exhibit well-dispersed nanoparticles, which can help them to be taken up by cells more easily than aggregated ones [8]. As expected, unlike pure PEG-POSS nanoparticles, the size of insulin-loaded PEG-POSS nanoparticles clearly increased as increasing $\mathrm{pH}$ (loading content $\sim 0.9 \mathrm{mg}$, Figure 3(b)).

\subsection{Insulin-Release Study}

Figure 4 shows insulin release behavior from insulinloaded PEG-POSS nanoparticles at different $\mathrm{pH}$ values of 2.0 and 7.4. At $\mathrm{pH}=2.0$, even though a tiny release of insulin was observed, probably due to a weakly physicaladsorbed insulin, PEG-POSS nanoparticles appeared to have higher insulin retention capacity at low $\mathrm{pH}$. Afterwards, following a $\mathrm{pH}$ change to 7.4 , a dramatic release of insulin was observed during the first hour followed by a relatively sustained release, due to a fast dissociation of insulin from PEG-POSS nanoparticles at higher $\mathrm{pH}$. This suggests that insulin was well-protected inside PEG-POSS nanoparticles at gastric $\mathrm{pH}$ for $2 \mathrm{hrs}$, and was released at intestinal $\mathrm{pH}(\mathrm{pH}$ 6-7) where the absorption and activation of the drug are necessary. We believe that such PEG-POSS nanoparticles could be used as a potential carrier for insulin drug delivery systems. The release data have been further studied [22] by fitting the cumulative fraction release data, $M_{t} / M_{\infty}$ to an empirical Equation (1). The drug release behavior according to diffusion controlled mechanism is usually governed by the following Equation (3),

$$
M_{t} / M_{\infty}=k t^{n}
$$

where $M_{\infty}$ is the total amount of insulin in dosage form, 


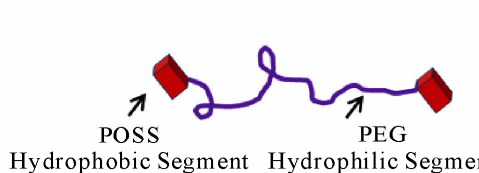

PEG-POSS telechelic

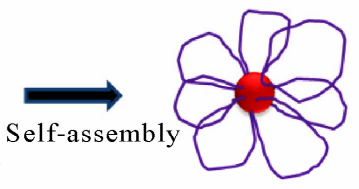

Flower like micelles

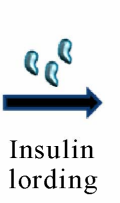

lording

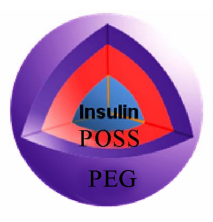

Core-shell type PEG3.4k-POSS NPs

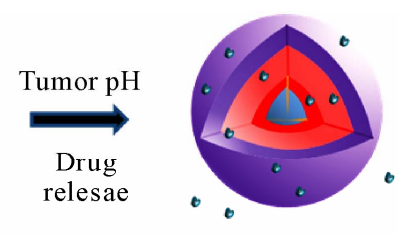

Scheme 1. Schematic illustration of core-shell nanostructured PEG-POSS nanoparticles loaded with insulin.
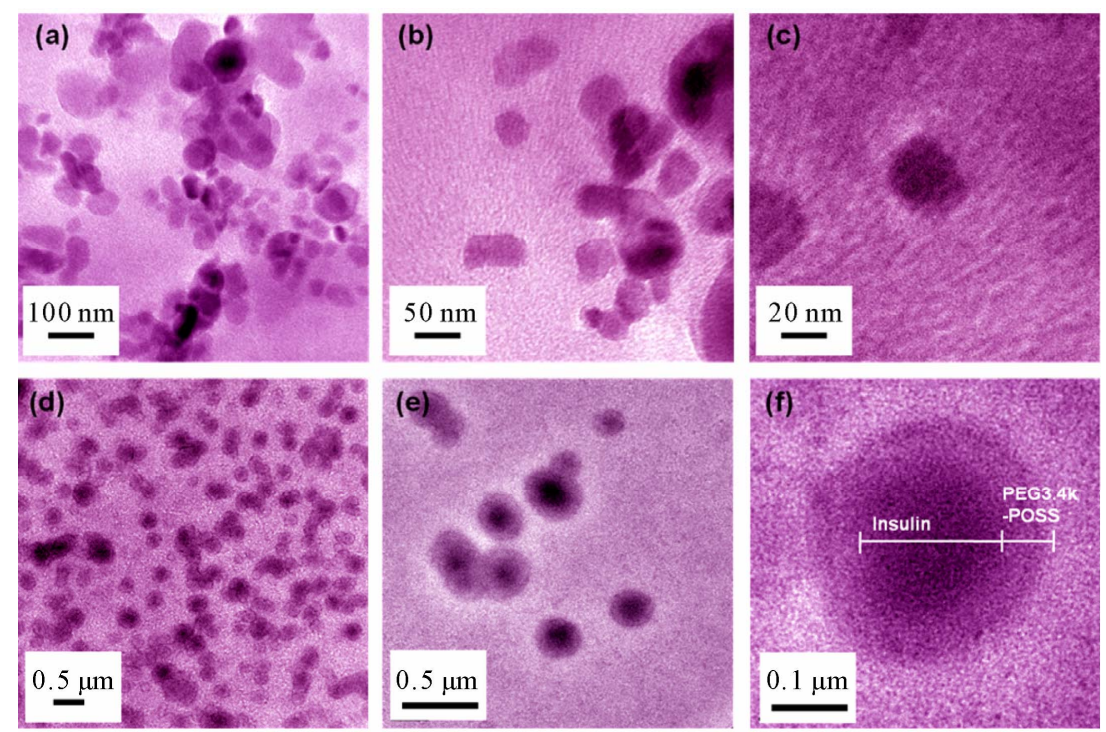

Figure 1. TEM images of pure (a, b, c) and insulin-loaded (d, e, f) PEG-POSS nanoparticles at different magnifications.

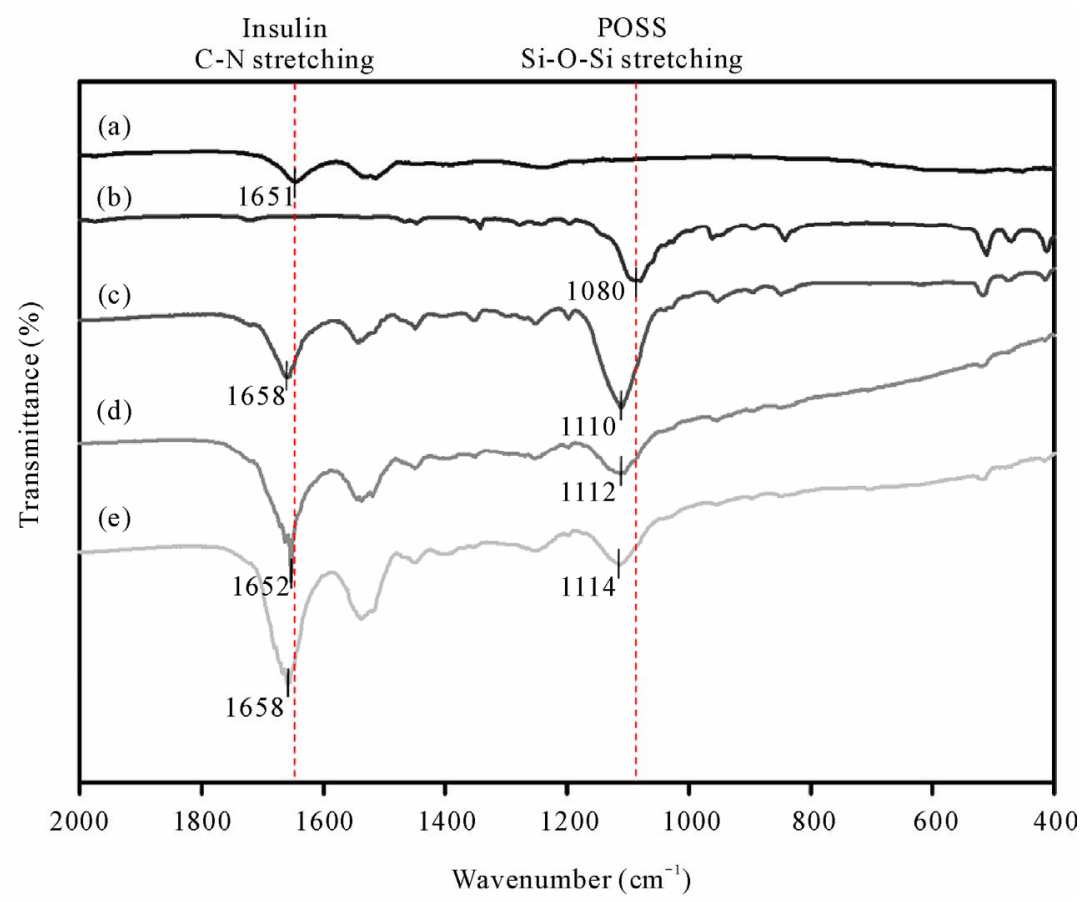

Figure 2. FTIR spectra of (a) pristine insulin, (b) pure PEG-POSS nanoparticles, and insulin-loaded PEG-POSS nanoparticles with different amount of insulin of (c) 0.5 , (d) 0.9 and (e) $1.3 \mathrm{mg}$. 


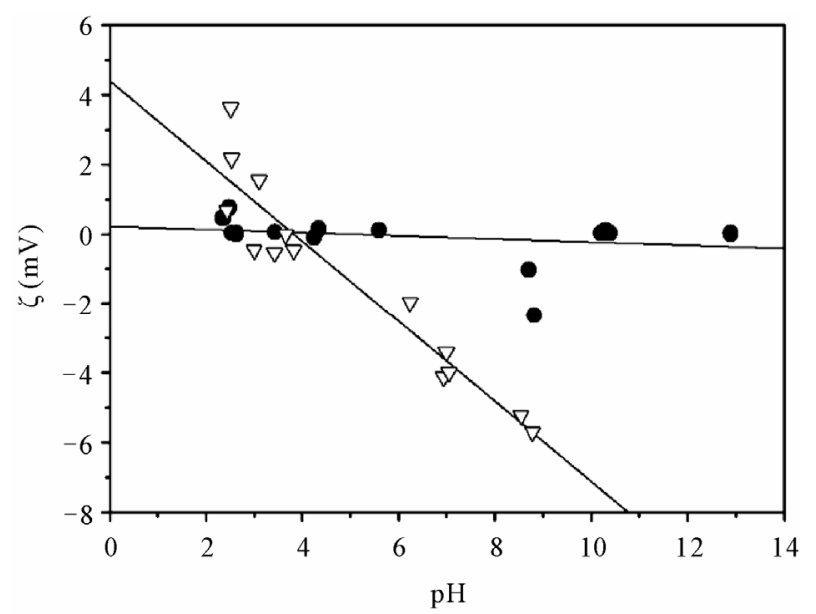

(a)

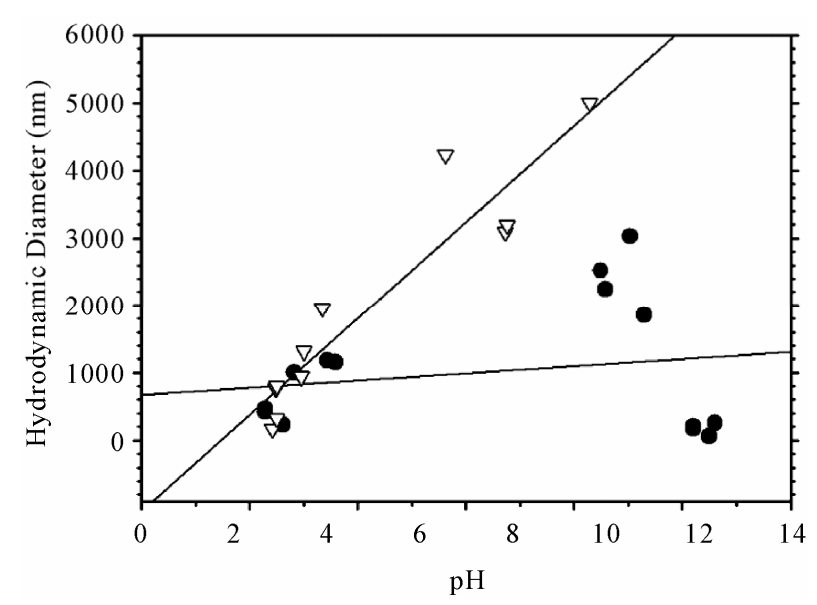

(b)

Figure 3. Zeta-potential (a) and particle size (b) of pure (•) and insulin-loaded $(\nabla)$ PEG-POSS nanoparticles with respect to the $\mathrm{pH}$.

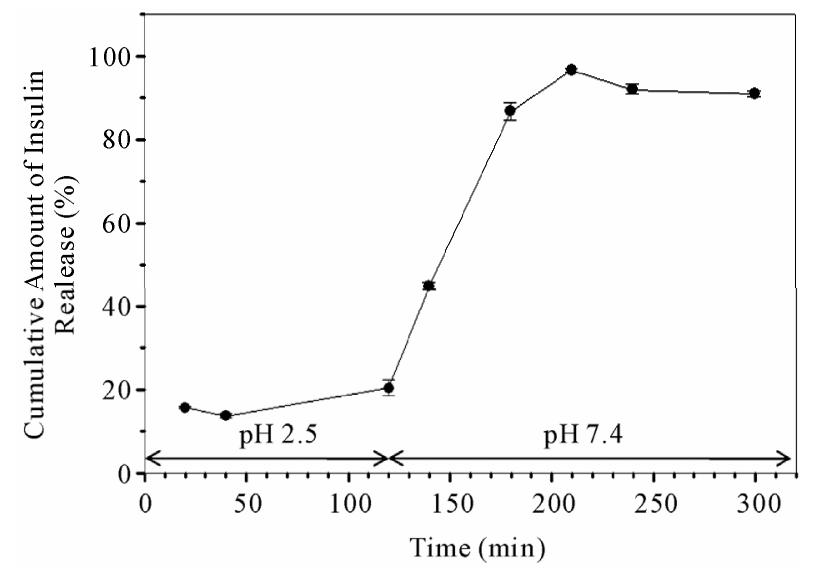

Figure 4. Insulin release from insulin-loaded PEG-POSS nanoparticles produced with an insulin mass of $0.9 \mathrm{ml}$ in gastric pH 2 simulated fluids for 2 hrs followed by additional 16 hrs in intestinal pH 7.4 simulated fluids at $37^{\circ} \mathrm{C}$.
$M_{t}$ is the amount of insulin released at time $t, k$ is kinetic constant, and $\mathrm{n}$ is diffusion or release exponent constant. Using the least-squares procedure, $n$ value was estimated to about 0.87 , suggesting anomalous diffusion or nonfickian diffusion. This finding refers to combination of both diffusion and erosion controlled rate release. Accordingly, it is expected that insulin release from PEOPOSS nanostructured nanoparticles was $\mathrm{pH}$-controlled, accompanying the swelling of insulin-loaded PEG-POSS nanoparticles.

\section{Conclusions}

We have successfully prepared the pure and insulinloaded nanostructured core-shell poly(ethylene glycol) (PEG)-polyhedral oligosilsesquioxane (POSS) nanoparticles via self-assembly process. TEM analysis demonstrated that pure and insulin-loaded self-assembled PEGPOSS nanoparticles were of spherical shape with coreshell nanostructure, and were well-dispersed and uniform in size distribution. Such PEG-POSS nanoparticles showed a good loading capability of hydrophobic drug, insulin. It was found that insulin was well-protected inside PEG-POSS nanoparticles at gastric $\mathrm{pH}$ for $2 \mathrm{hrs}$, and was released at intestinal $\mathrm{pH}(\mathrm{pH} 6$ - 7) where the absorption and activation of the drug are necessary. As a result, insulin release from PEG-POSS nanoparticles was pH-dependent.

\section{Acknowledgements}

The authors acknowledge the support of Shinshu University Global COE Program "International Center of Excellence on Fiber Engineering". This paper is dedicated to the first principal, Chotaro Harizuka, on the occasion of 100th anniversary of Faculty of Textile Science and Technology, Shinshu University

\section{REFERENCES}

[1] J. J. Shi, A. R. Votruba, O. C. Farokhzad and R. Langer, "Nanotechnology in Drug Delivery and Tissue Engineering: From Discovery to Applications," Nano Letters, Vol. 10, No. 9, 2010, pp. 3223-3230. doi: $10.1021 / \mathrm{nl} 102184 \mathrm{c}$

[2] J. H. Kwon, B. H. Lee, J. J. Lee and C. W. Kim, "Insulin Microcrystal Suspension as a Long-Acting Formulation for Pulmonary Delivery," European Journal of Pharmaceutical Science, Vol. 22, No. 2-3, 2004, pp. 107-116. doi:10.1016/j.ejps.2004.02.007

[3] M. Goldberg and I. Gomez-Orellana, "Challenges for the Oral Delivery of Macromolecules," Nature Reviews Drug Discovery, Vol. 2, No. 4, 2003, pp. 289-295. doi: $10.1038 / \mathrm{nrd} 1067$

[4] B. S. Kim and P. T. Mather, "Amphiphilic Telechelics Incorporating Polyhedral Oligosilsesquioxane: 1. Synthesis 
and Characterization," Macromolecules, Vol. 35, No. 22, 2002, pp. 8378-8384. doi:10.1021/ma020226h

[5] B. S. Kim and P. T. Mather, "Morphology, Microstructure, and Rheology of Amphiphilic Telechelics Incorporating Polyhedral Oligosilsesquioxane," Macromolecules, Vol. 39, No. 26, 2006, pp. 9253-9260. doi:10.1021/ma062069i

[6] W. J. Lee, S. Ni, J. Deng, B. S. Kim, S. K. Satija, P. T. Mather and A. R. Esker, "Telechelic Poly(ethylene glycol)-POSS Amphiphiles at the Air/Water Interface," Macromolecules, Vol. 40, No. 3, 2007, pp. 682-688. doi:10.1021/ma0618171

[7] B. S. Kim and P. T. Mather, "Amphiphilic Telechelics with Polyhedral Oligosilsesquioxane(POSS) End-groups: Dilute Solution Viscometry," Polymer, Vol. 47, No. 17, 2006, pp. 6202-6207. doi:10.1016/j.polymer.2006.06.050

[8] C. K. Kim, B. S. Kim, F. A. Sheikh, U. S. Lee, M. S. Khil and H. Y. Kim, "Amphiphilic Poly(Vinyl Alcohol) Hybrids and Electrospun Nanofibers Incorporating Polyhedral Oligosilsesquioxane," Macromolecules, Vol. 40, No. 14, 2007, pp. 4823-4828. doi:10.1021/ma070056e

[9] F. A. Sheikh, N. A. M. Barakat, B. S. Kim, S. Aryal, M. S. Khil and H. Y. Kim, "Self-Assembled Amphiphilic Polyhedral Oligosilsesquioxane(POSS) Grafted Poly(Vinyl Alcohol) (PVA) Nanoparticles," Materials Science and Engineering C, Vol. 29, No. 3, 2009, pp. 869-876. doi:10.1016/j.msec.2008.07.029

[10] W. Zhang, B. Fang, A. Walther and A. H. E. Muller, "Synthesis via RAFT polymerization of Tadpole-shaped Organic/inorganic Hybrid PAA Containing POSS and Their Self-Assembly in Water," Macromolecules, Vol. 42, No. 7, 2009, pp. 2563-2569. doi:10.1021/ma802803d

[11] W. Zhang, L. Liu, X. D. Zhuang, X. Li, J. Bai and Y. Chen, "Synthesis and Self-assembly of Tadpole-Shaped Organic/ inorganic Hybrid Poly(N-isopropylacrylamide) Containing Polyhedral Oligomeric Silsesquioxane via RAFT Polymerization," Journal of Polymer Science Part A: Polymer Chemistry, Vol. 46, No. 21, 2008, pp. 7049-7061. doi:10.1002/pola.23010

[12] H. Hussain, B. H. Tan, G. L. Seah, Y. Liu, C. B. He and T. P. Davis, "Micelle Formation and Gelation of (PEGP(MA-POSS)) Amphiphilic Block Copolymers via Associative Hydrophobic Effects," Langmuir, Vol. 26, No. 14, 2010, pp. 11763-11773. doi:10.1021/la101686q

[13] B. H. Tan, H. Hussain and C. B. He, "Tailoring Micelle Formation and Gelation in (PEG-P(MA-POSS)) Amphi- philic Hybrid Block Copolymers," Macromolecules, Vol. 44, No. 3, 2011, pp. 622-631. doi:10.1021/ma102510u

[14] K. O. Kim, Y. A. Seo, B. S. Kim, K. J. Yoon, M. S. Khil, H. Y. Kim and I. S. Kim, "Transition Behaviors and Hybrid Nanofibers of Poly(vinyl alcohol) and polyethyleneglycol-POSS Telechelic Blends," Colloid and Polymer Science, Vol. 289, No. 8, 2011, pp. 863-870. doi:10.1007/s00396-011-2407-y

[15] Y. H. Lin, F. L. Mi, C. T. Chen, W. C. Chang, S. F. Peng, H. F. Liang and H. W. Sung, "Preparation and Characterization of Nanoparticles Shelled with Chitosan for Oral Insulin Delivery," Biomacromolecules, Vol. 8, No. 1, 2007, pp. 146-152. doi:10.1021/bm0607776

[16] Y. H. Lin, C. T. Chen, H. F. Liang, A. R. Kulkarni, P. W. Lee, C. H. Chen and H. W. Sung, "Novel Nanoparticles for Oral Insulin Delivery via the Paracellular Pathway," Nanotechnology, Vol. 18, No. 10, 2007, pp. 105-102. doi:10.1088/0957-4484/18/10/105102

[17] V. Sluzky, J. A. Tamada, A. M. Klibanovt and R. Langer, "Kinetics of Insulin Aggregation in Aqueous Solutions upon Agitation in the Presence of Hydrophobic Surfaces," Applied Biological Sciences, Vol. 88, No. 21, 1991, pp. 9377-9381.

[18] C. L. Lawson and R. J. Hanson, "Solving Least Squares Problem," Prentice-Hall, Englewood Cliffs, 1974.

[19] B. Sarmento, A. Ribeiro, F. Veiga, D. Ferreira and R. Neufeld, "Oral Bioavailability of Insulin Contained in Polysaccharide Nanoparticles," Biomacromolecules, Vol. 8, No. 10, 2007, pp. 3054-3060. doi:10.1021/bm0703923

[20] K. Sonaje, K. J. Lin, J. J. Wang, F. L. Mi, C. T. Chen, J. H. Juang and H. W. Sung, "Self-Assembled pH-Sensitive Nanoparticles: A platform for Oral Delivery of Protein Drugs," Advanced Functional Materials, Vol. 20, No. 21, 2010, pp. 3695-3700. doi:10.1002/adfm.201001014

[21] S. Gelinas, C. Chapados, M. Beauregard, I. Gosselin and M. G. Martinoli, "Effect of Oxidative Stress on Stability and Structure of Neurofilament Proteins," Biochemistry and Cell Biology, Vol. 78, No. 6, 2000, pp. 667-674. doi: $10.1139 / 000-070$

[22] W. P. Wang, X. X. Jie, M. Fei and H. Jiang, "Synthesis of Core-shell Particles by Batch Emulsion Polymerization of Styrene and Octavinyl Polyhedral Oligomeric Silsesquioxane," Journal of Polymer Reseraches, Vol. 18, No. 1, 2011, pp. 13-17. doi:10.1007/s10965-009-9385-5 